ARTICLE

DOI: $10.1038 / s 41467-018-04023-z$

OPEN

\title{
Spontaneous formation of gold nanostructures in aqueous microdroplets
}

\author{
Jae Kyoo Lee ${ }^{1}$, Devleena Samanta ${ }^{1}$, Hong Gil Nam (iD ${ }^{2,3}$ \& Richard N. Zare (i) ${ }^{1}$
}

The synthesis of gold nanostructures has received widespread attention owing to many important applications. We report the accelerated synthesis of gold nanoparticles (AuNPs), as well as the reducing-agent-free and template-free synthesis of gold nanoparticles and nanowires in aerosol microdroplets. At first, the AuNP synthesis are carried out by fusing two aqueous microdroplet streams containing chloroauric acid and sodium borohydride. The AuNPs ( $7 \mathrm{~nm}$ in diameter) are produced within $60 \mu$ s at the rate of $0.24 \mathrm{~nm}_{\mu} \mathrm{s}^{-1}$. Compared to bulk solution, microdroplets enhance the size and the growth rate of AuNPs by factors of about 2.1 and $1.2 \times 10^{5}$, respectively. Later, we find that gold nanoparticles and nanowires $(\sim 7$ $\mathrm{nm}$ wide and $>2000 \mathrm{~nm}$ long) are also formed in microdroplets in the absence of any added reducing agent, template, or externally applied charge. Thus, water microdroplets not only accelerate the synthesis of AuNPs by orders of magnitude, but they also cause spontaneous formation of gold nanostructures.

\footnotetext{
${ }^{1}$ Department of Chemistry, Stanford University, Stanford, CA 94305, USA. ${ }^{2}$ Center of Plant Aging Research, Institute for Basic Science, Daegu 42988, Republic of Korea. ${ }^{3}$ Department of New Biology, DGIST, Daegu 42988, Republic of Korea. Correspondence and requests for materials should be addressed to H.G.N. (email: nam@dgist.ac.kr) or to R.N.Z. (email: zare@stanford.edu)
} 
G old nanostructures are one of the most widely studied materials owing to the unique properties of nanoscale gold such as quantum size effects, surface plasmon resonance, high catalytic activity, self-assembly, etc. ${ }^{1-5}$. The synthesis of gold nanoparticles (AuNPs) typically takes seconds to hours ${ }^{6,7}$. In many cases, capping agents are added because they stabilize the nanoparticles and also act as structure-directing agents, leading to the formation of anisotropic nanostructures such as gold nanowires (AuNWs), nanoplates, nanoprisms, etc. ${ }^{8,9}$. Alternatively, the templates can be used for the anisotropic growth of gold nanostructures ${ }^{10-12}$. Control over both size and shape of the nanostructures is important as both factors strongly influence optical, mechanical, electrical, and chemical properties of the nanostructures.

Recently, microdroplet chemistry has emerged as a unique platform for studying and performing chemical reactions. Microdroplet fusion mass spectrometry, developed by our group previously, allows the capture of events occurring on the microsecond timescale ${ }^{13}$. It has been found that chemical reactions in micron-sized liquid droplets (microdroplets) exhibit unusual reaction properties that are not observed in bulk solutions; in particular, some reaction rates can be enhanced by factors of $10^{3}-10^{6} 13-27$. All reactions explored to date are simple bimolecular reactions. We report the crystallization kinetics of gold precursors in the microdroplets. Our objective was to study the formation and growth of AuNPs in microdroplets and whether nanocrystallization in the microdroplets is different from that in the bulk solution. Accelerated crystallization and subsequent nanoparticle formation are fundamentally different from bimolecular reactions, as it requires fast reaction and coalescence of hundreds of individual species within a span of microseconds.

There have been several studies on nanoparticle synthesis using micron-sized reactors (e.g., using microfluidic platforms), mostly aimed at precise manipulation of growth, rapid mixing, fast timeresolved analysis, and multiple phase synthesis ${ }^{28,29}$. However, the controllable timescale for growth or analysis using the microreactors is only limited to tens of milliseconds with sub-second dead times ${ }^{30}$. Another approach using microreactors for nanoparticle synthesis is spraying the charged droplets containing precursor metal ions onto a grounded cathode surface, in which the metal nanoparticles are formed on the cathode surface ${ }^{31}$. This study did not provide information on the kinetics of the process.

In contrast to these previous approaches, we used high-speed fused aerosol microdroplets for nanoparticle formation, allowing observation with microsecond temporal resolution. We followed a well-established procedure, where $\mathrm{AuCl}_{4}{ }^{-}$ions coming from chloroauric acid $\left(\mathrm{HAuCl}_{4}\right)$ are reduced by sodium borohydride $\left(\mathrm{NaBH}_{4}\right)^{32,33}$. $\mathrm{NaBH}_{4}$ is a classic reductant that plays dual roles of reducing gold ions and stabilizing AuNPs against aggregation ${ }^{32}$.

We investigate the kinetics of AuNP formation by fusing the two microdroplets, each containing one reactant, and varying the traveling distance of the fused microdroplets to the collection device. As a control, we also examine what happens when we use aqueous microdroplets containing no added reducing agent. We find that the microdroplets are also effective in producing AuNPs and AuNWs in the absence of any reducing agent and template.

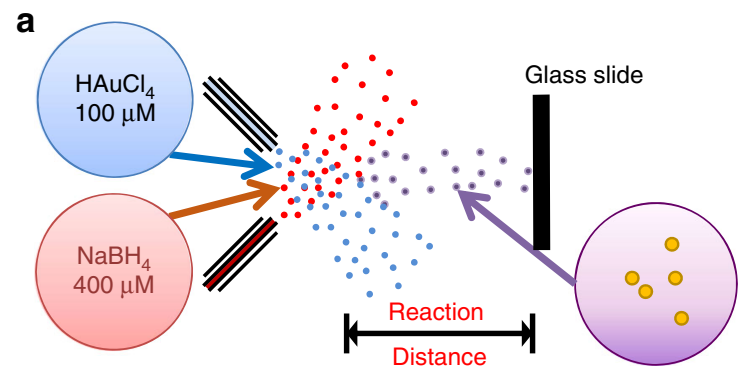

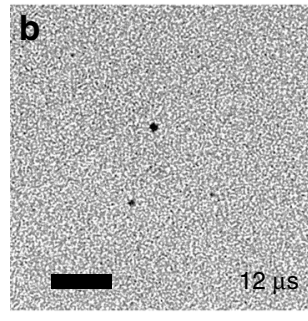

C

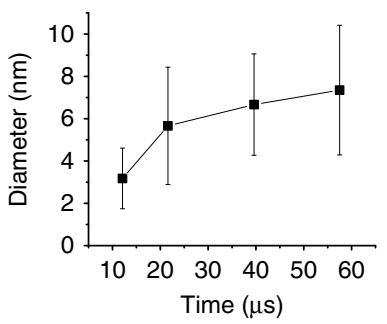

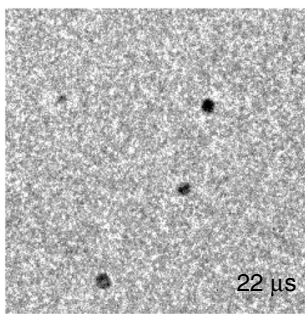
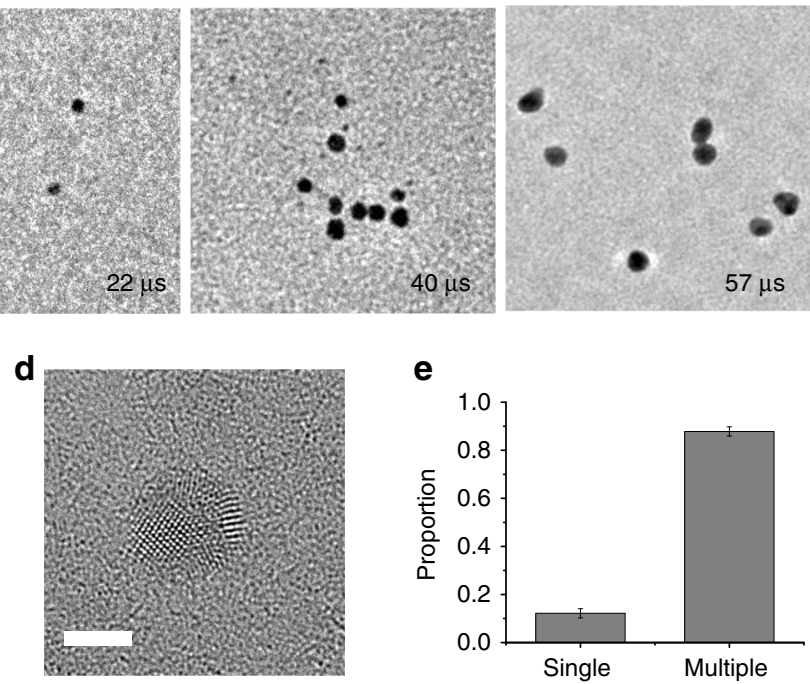

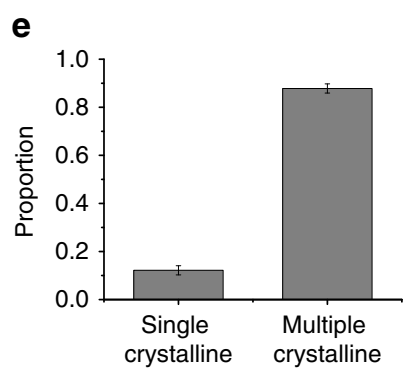

Fig. 1 Kinetically controlled AuNP growth using fused microdroplets. a Schematic of the experiment setup. Microdroplets containing $100 \mu \mathrm{M} \mathrm{HACl}_{4}$

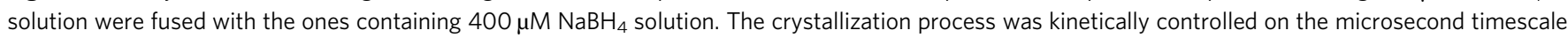
by adjusting the traveling distance of the fused microdroplets. b TEM images of gold nanoparticles at different time points. Scale bar is 20 nm. $\mathbf{c}$ Timecourse change in the diameter of the gold nanoparticles. $\mathbf{d}$ TEM image of a nanoparticle showing multiple crystalline structures. e Portion of nanoparticles with single or multiple crystalline structures synthesized in microdroplets. Error bars represent one standard deviation of three replicates 
We also find that this occurred in microdroplets without any added reducing agents or externally applied charges. The spontaneous formation of nanostructures is attributed to the entropic changes that overcome the free-energy barrier in the reaction, caused in part by a strong electric field at the water-air interface of the microdroplets. Our results further reveal the unique properties of the microdroplets and provide a new method for a highly efficient synthesis of nanostructures under benign, environmentally friendly, and ambient conditions.

\section{Results}

Kinetically controlled growth of AuNPs in microdroplets. Figure la shows a schematic of the experimental setup. A stream of aqueous microdroplets containing $100 \mu \mathrm{M} \mathrm{HAuCl}$ solution was fused with a stream of aqueous microdroplets containing 400 $\mu \mathrm{M} \mathrm{NaBH}$ solution. Unlike previous studies with fused microdroplets, no voltage was applied to either of the streams. The reaction progressed as the fused microdroplets traveled toward a glass slide where they were collected. The reaction time was adjusted by varying the traveling distance. The estimated reaction time was calculated by integrating the speed of the microdroplets over the traveling distance, as previously described ${ }^{13}$. The size of a fused aqueous microdroplet remained almost constant within the observation time frame, showing that almost minimal evaporation occurred in the microdroplets during their traveling time $^{13,15}$.

Figure $1 \mathrm{~b}$ shows representative transmission electron microscope (TEM) images of the synthesized AuNPs at different reaction times $(12-57 \mu \mathrm{s})$. Exposure time and intensity of the ebeam for TEM imaging were minimized to avoid sintering or modification of the nanoparticle structure. Figure 1c presents the time-course change in the averaged diameter of AuNPs. We observe that within as early as $12 \mu \mathrm{s}$, particles as large as $3 \mathrm{~nm}$ are formed. The rate of the nanoparticle growth was calculated to be $0.24 \mathrm{~nm} \mu \mathrm{s}^{-1}$. The reaction notably slowed down after $\sim 21 \mu \mathrm{s}$, although the reaction did not reach equilibrium within the observation time frame. The mean diameter of nanoparticles at $57 \mu$ s was $\sim 7 \mathrm{~nm}$. Our results indicate that nanoparticle formation involves fast nucleation followed by slower growth. These results are consistent with the mechanism put forward by Emmerling and co-workers, who measured the growth rate of AuNPs by mixing $\sim 500 \mu \mathrm{M} \mathrm{HAuCl}_{4}$ and $2 \mathrm{mM} \mathrm{NaBH}_{4}$ solutions, flowing the mixed solution in a 1-mm size channel, and monitoring the growth kinetics with on-line small-angle X-ray scattering ${ }^{34}$. They observed that nucleation occurred within $200 \mathrm{~ms}$, the resolution limit of their set up. The growth rate of nanoparticles in the bulk solution was $\sim 0.03 \mathrm{~nm} \mathrm{~s}^{-}$. It took several hours for the reaction to reach equilibrium. In comparison, we find that in microdroplets, the initial nucleation events occur at least $2.8 \times 10^{4}$ fold faster. Moreover, the growth of AuNPs in the microdroplets was enhanced by a factor of $1.2 \times 10^{5}$, compared to bulk solution, even considering the higher concentrations of precursors used for the reaction in bulk continuous flow. Comparing the size of AuNPs between the microdroplet and bulk solution after the reaction significantly slowed down, the AuNP size in the microdroplet was $\sim 2.1$ times larger than that in the bulk solution. A high-resolution TEM image (Supplementary Fig. 1) shows the indexing of the two-dimensional lattice fringes to be $\sim 2.3 \AA$, corresponding to the lattice spacing of $\mathrm{Au}$ (111). This confirms that the nanoparticles have the crystal structure of pure Au.

Figure 1d, e shows a TEM image of a representative nanoparticle with multiple crystalline structure and the relative proportion of single and multiple crystalline structures. It is not clear whether the multiple crystalline structures were formed by smaller single crystalline nanoparticles coalescing or by the
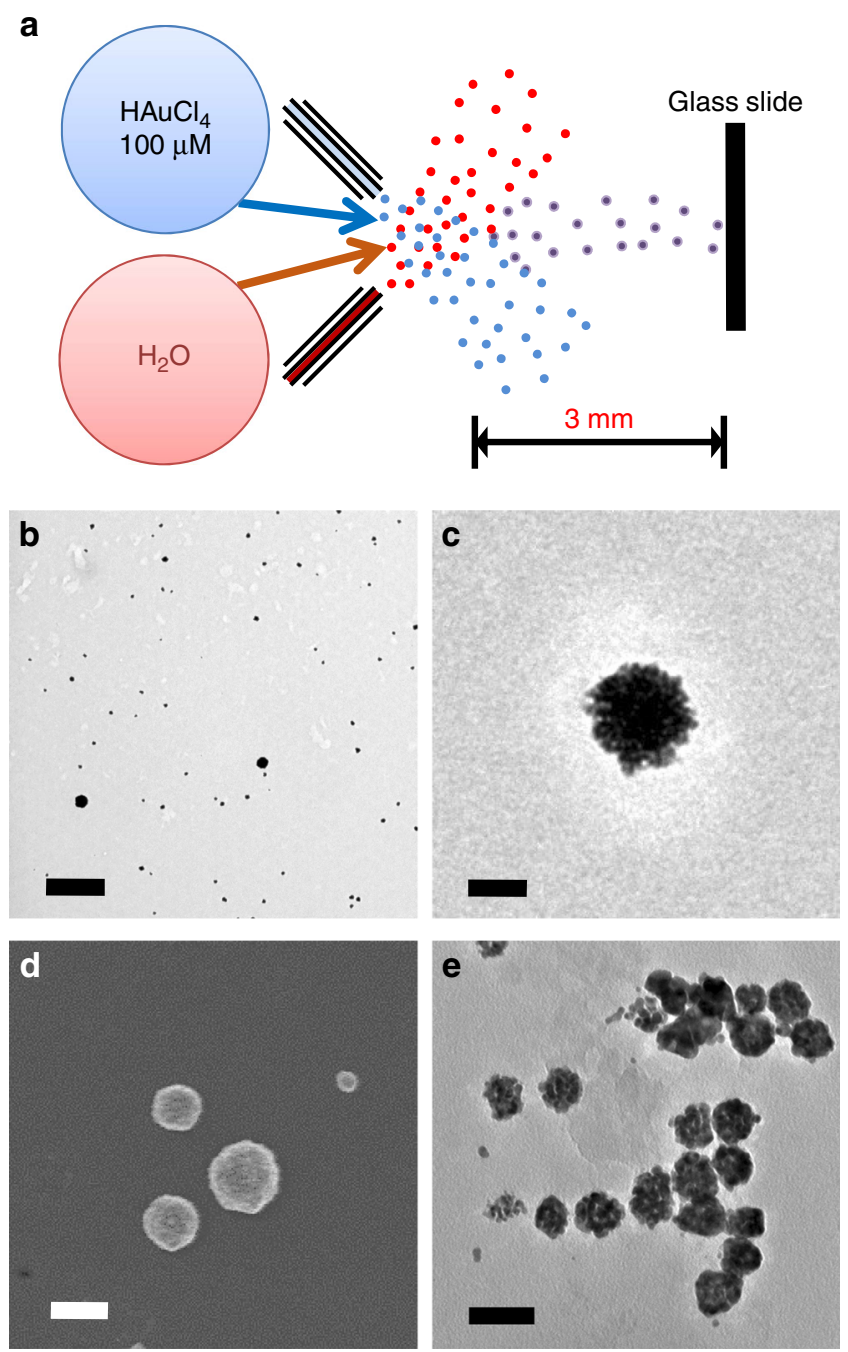

Fig. 2 Reducing-agent-free synthesis of AuNPs in the microdroplets with no externally applied charges. a schematic of the experimental setup for reducing-agent-free synthesis of AuNP in the microdroplets. Scale bar is 1 $\mu \mathrm{m}$. b, c TEM images of the synthesized AuNPs and AuNP aggregates. d SEM image of the AuNP aggregates. e TEM image showing an intermediate aggregated status of AuNP aggregates. Scale bars for b-e are $50 \mathrm{~nm}$

inhomogeneous environment of the microdroplet. Further kinetic studies at higher temporal resolution with a slower reaction rate, for example, by lowering the growth temperature or the precursor concentration, may reveal what mechanism is responsible for the multiple crystalline structures. To date, we have not succeeded in sufficiently slowing down the crystallization process to acquire this information.

We confirmed that most of the AuNP growth occurred in microdroplets rather than on the surface of the glass slide or during the transfer to a TEM grid. This was achieved by examining the average diameter of nanoparticles under two different drying conditions. The size of AuNPs prepared by air drying for $30 \mathrm{~min}$ after collecting the aqueous microdroplets containing nanoparticles are not significantly different from the size of the nanoparticles prepared in a vacuum desiccator for 5 min (Supplementary Fig. 2).

AuNP formation in reducing-agent-free microdroplets. As a control experiment, we fused the aqueous droplets containing $100 \mu \mathrm{M} \mathrm{HAuCl}_{4}$ with the aqueous microdroplets (Fig. 2a) without any externally added charges. The fused microdroplets containing 

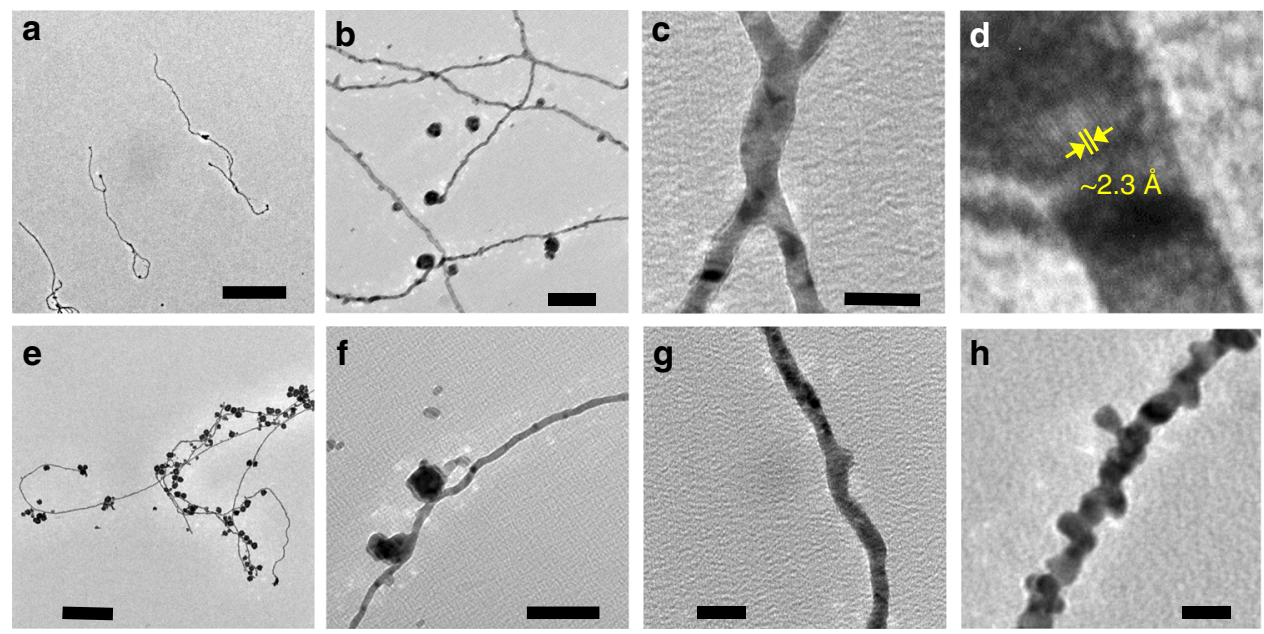

Fig. 3 Reducing-agent-free and template-free synthesis of Au nanowires (AuNWs) in the aqueous microdroplets. a AuNWs with several micrometer length. Scale bar is $1 \mu \mathrm{m}$. b Formation of AuNW network. Scale bar is $100 \mathrm{~nm}$. c Junction between two AuNWs. Scale bar is $20 \mathrm{~nm}$. d A TEM image showing the lattice spacing of $\sim 2.3 \AA$, corresponding to the Au(111) structure, confirming that the nanowire is made of pure Au. e, $\mathbf{f} A u N W s$ accompanying with AuNP and AuNP aggregates. Scale bars for $\mathbf{e}$ and $\mathbf{f}$ are $300 \mathrm{~nm}$ and $100 \mathrm{~nm} . \mathbf{g}$, h Two different types of AuNWs formed in the microdroplets with a smooth surface $(\mathbf{g})$ and beaded surface (h). Scale bars for $\mathbf{g}$ and $\mathbf{h}$ are $20 \mathrm{~nm}$

only $\mathrm{HAuCl}_{4}$ solution were collected after $3 \mathrm{~mm}$ of traveling for $\sim 40 \mu$ s traveling time. Surprisingly, we have found the formation of AuNPs, as evidenced by the TEM image in Fig. 2b. Closer inspection revealed that in addition to small nanoparticles $(\sim 7 \mathrm{~nm}$ in diameter), larger particles ( $\sim 30 \mathrm{~nm}$ in diameter), caused by non-specific aggregation of the AuNPs, were also formed (Fig. 2c). The identity of the AuNPs was verified by energy dispersive spectroscopy (EDS) (Supplementary Fig. 3) as well as by measuring the lattice spacing. A high-resolution SEM image confirmed that the shapes of the AuNP particles are spherical (Fig. 2d). Figure 2e presents a TEM image showing both individual AuNPs and AuNP aggregates, as well as half-way aggregated intermediate forms that illustrates how the AuNP aggregates were formed.

We examined whether inelastic collisions of the microdroplets, which provide kinetic energy, may contribute to the nanoparticle formation. We used only one stream of microdroplet containing $100 \mu \mathrm{M} \mathrm{HAuCl}{ }_{4}$ solution without fusing with other microdroplets. The microdroplets traveling for $3 \mathrm{~mm}$ were collected on a glass slide. Similar nanoparticle or nanoparticle-aggregate formation was observed using single stream of microdroplets, suggesting that the nanoparticle formation in the microdroplets was not caused by collisional activation. The nanoparticle formation was further verified by collecting the $\mathrm{HAuCl}_{4}$ microdroplets at $3 \mathrm{~mm}$ distance from the stream over a period of 30 min and carrying out dynamic light scattering analysis.

We also ruled out the possibility of unintended formation of AuNPs by the e-beam irradiation used for TEM imaging, as the ebeam can produce and manipulate metal nanoparticles ${ }^{35-37}$. Supplementary Figure 4 shows two TEM images taken before and after a continuous e-beam exposure for $20 \mathrm{~min}$. No difference in the structure of the nanoparticles or no new formation of nanoparticles or any other nanostructure confirms that the nanoparticles were not synthesized by the e-beam used for the TEM imaging, rather the effect of microdroplets. We also ascertained that AuNPs were not formed through the physical contact with metal substrates in the TEM grids by examining the TEM grids made from different materials.

To explore the mechanism of the AuNP formation in the absence of any reducing agent, we carried out mass spectrometric analysis of $\mathrm{HAuCl}_{4}$ solution in the microdroplets. A $10 \mu \mathrm{M}$ $\mathrm{HAuCl}_{4}$ solution was sprayed in forms of microdroplets. The travel distance of microdroplets was $1.5 \mathrm{~cm}$ that allows the analytes to be exposed to the microdroplet environment for $\sim 180$ $\mu \mathrm{s}$. Supplementary Figure 5 shows a mass spectrum of the $\mathrm{HAuCl}_{4}$ solution in the microdroplets. The original form of gold, $\mathrm{AuCl}_{4}{ }^{-}$, with an oxidation number +3 , as well as the reduced forms of gold, $\mathrm{Au}_{2} \mathrm{Cl}_{3}^{-}$(oxidation number +1 ), $\mathrm{AuCl}_{2}{ }^{-}$ (oxidation number +1 ), and $\mathrm{Au}_{2} \mathrm{Cl}_{4}{ }^{-}$(oxidation number +1.5 ) were observed. Supplementary Table 1 summaries the gold molecular species observed in the microdroplets containing $\mathrm{HAuCl}_{4}$ solution. We also examined the possibility of thermal decomposition of $\mathrm{AuCl}_{4}{ }^{-}$ions ${ }^{38}$ that can be possibly caused due to the high temperature at the mass spectrometer capillary inlet $\left(275^{\circ} \mathrm{C}\right)$. We have decreased the temperature of the capillary inlet down to $60^{\circ} \mathrm{C}$, below the typical thermal decomposition temperature. Essentially, the same mass spectrum was acquired with spontaneous reduction of gold ions at the lowered temperature. These results present direct evidence that gold ions were reduced spontaneously to form AuNPs in the microdroplets without an added reducing agent.

AuNW growth in droplets free of reducing agent and template. AuNWs can be synthesized by various methods including chemical, electrochemical, and epitaxial routes ${ }^{39,40}$. The formation often needs structure-directing capping agents ${ }^{41}$ or porous templates that guide their directional growth ${ }^{42}$. However, we find that AuNWs $(\sim 7 \mathrm{~nm}$ wide) can be formed in the microdroplets without using any template or reducing agent, as well as externally applied charges (Fig. 3). The average length of AuNWs formed in aqueous microdroplets was $2195 \mathrm{~nm}$, raging from hundreds of nanometer to approximately six micrometers (Fig. 3a). Figure 3b, c shows that not only single-strand wires, but also a network of nanowires appeared. The constitution of the nanowires with pure $\mathrm{Au}$ was confirmed by the lattice spacing of $\sim 2.3 \AA$, matching with the Au (111) structure (Fig. 3d), and EDS analysis (Supplementary Fig. 6).

A form of nanowires accompanied with AuNP or AuNP aggregates were also observed (Fig. 3e, f). This nanowire and nanoparticle junction can potentially be used for remote excitation of surface-enhanced Raman scattering ${ }^{43}$, catalysis, or physical entrapment of materials ${ }^{44}$. Two different forms of AuNWs were found in the microdroplets: one with a relatively smooth surface (Fig. 3g) and the other with a beaded surface 


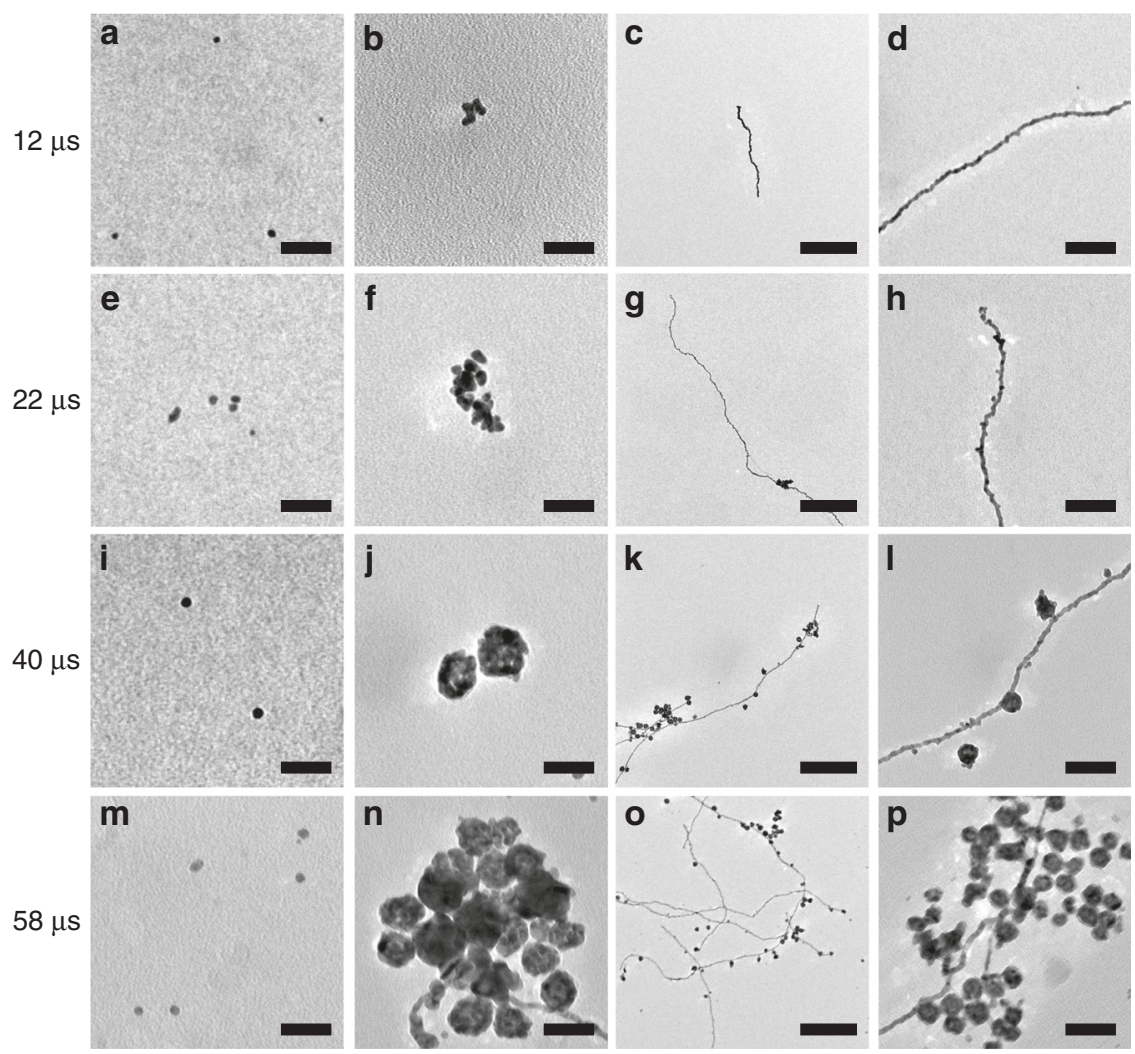

Fig. 4 TEM images at different time points of AuNPs and AuNWs grown in the aqueous microdroplets. Time-course changes in the individual AuNPs (a, e, $\mathbf{i}, \mathbf{m})$, AuNP aggregates $(\mathbf{b}, \mathbf{f}, \mathbf{j}, \mathbf{n})$, and AuNWs ( $\mathbf{c}, \mathbf{d}, \mathbf{g}, \mathbf{h}, \mathbf{k}, \mathbf{I}, \mathbf{0}, \mathbf{p})$. Scale bars for $\mathbf{a}, \mathbf{b}, \mathbf{e}, \mathbf{f}, \mathbf{i}, \mathbf{j}, \mathbf{m}, \mathbf{n}$ are $40 \mathrm{~nm}$. Scale bars for $\mathbf{c}, \mathbf{g}, \mathbf{k}, \mathbf{0}$ are $80 \mathrm{~nm}$. Scale bars for $\mathbf{d}, \mathbf{h}, \mathbf{I}, \mathbf{p}$ are $80 \mathrm{~nm}$

(Fig. 3h). The beaded wires appears to be composed of a series of individual AuNPs. On the other hand, the nanowires with smooth surface do not appear to be made of individual or clusters of AuNPs, although there are multiple crystalline domains present.

Kinetics of spontaneous AuNP/AuNW growth in the microdroplets. We further studied the kinetics of the spontaneous AuNP and AuNW growth in the microdroplets. Aqueous microdroplets containing $100 \mu \mathrm{M} \mathrm{HAuCl}{ }_{4}$ solution were generated using high-pressure nebulizing dried $\mathrm{N}_{2}$ gas. The traveling distance between the point where the microdroplets were generated and the point where the microdroplets were collected was adjusted to control the exposure time of $\mathrm{Au}$ precursors to the microdroplet environment. The collected samples were transferred to a TEM for imaging analysis. Figure 4 shows the TEM time-series images of individual AuNP growth (Fig. 4a, e, i, m), AuNP aggregates (Fig. 4b, f, j, n), and AuNWs (Fig. 4c, d, g, h, k, $1, o, p)$. Figure $5 \mathrm{a}-\mathrm{c}$ shows the quantitative analysis of the timecourse changes in overall AuNP diameter (Fig. 5a), individual AuNP and AuNP aggregates (Fig. 5b), and the thickness of AuNW (Fig. 5c). Although the overall size of AuNP including individual AuNP and AuNP aggregates, increases (Fig. 5a), the size of an individual AuNP does not grow significantly (only up to $\sim 8 \mathrm{~nm}$, see Fig. $4 \mathrm{a}, \mathrm{e}, \mathrm{i}, \mathrm{m}$, and Fig. $5 \mathrm{~b}$ ). The increase of average AuNP size was mainly attributed to AuNP aggregates rather than the increase in individual AuNP size.

Interestingly, the thickness of AuNW decreased over time in the microdroplets, which was somewhat counterintuitive. There were two different types of AuNWs: the one with a smooth surface and the other with a beaded surface. The bimodal distribution of the thickness of AuNWs grown in the microdroplets is reflective of the size difference between the two types of AuNWs (Fig. 5d). The thickness of the smooth-surfaced AuNWs is centered at around $6 \mathrm{~nm}$, whereas the beaded AuNWs at around $14 \mathrm{~nm}$. The decrease in the thickness of the AuNW was because the portion of beaded AuNWs became smaller than that of the smooth AuNWs. It is still unclear what causes the relative proportion of the nanostructures to vary with time.

We have carried out experiments of spraying the fused microdroplets on a $\mathrm{NaBH}_{4}$-deposited glass slide to examine whether any further reaction can occur during the collection on a glass slide. Two separate types of experiments: (1) the fused microdroplets between the microdroplets containing $100 \mu \mathrm{M}$ $\mathrm{HAuCl}_{4}$ and the microdroplets containing $400 \mu \mathrm{M} \mathrm{NaBH}$, and (2) the fused microdroplets between the microdroplets containing $100 \mu \mathrm{M} \mathrm{HAuCl}_{4}$ and the aqueous microdroplets with no $\mathrm{NaBH}_{4}$. The traveling distance of the fused microdroplets to the $\mathrm{NaBH}_{4}$ desposited collector was $15 \mathrm{~mm}$. For the fused microdroplets containing both $\mathrm{HAuCl}_{4}$ and $\mathrm{NaBH}_{4}$, there was essentially no difference in the distribution of size of the synthesized nanoparticles (Supplementary Fig. 7). This suggests that the process of nanoparticle formation is almost completed in the microdroplets before reaching the collection slide. On the other hand, for the fused microdroplets between only $\mathrm{HAuCl}_{4}$ and water microdroplets, more nanoparticles with $<10 \mathrm{~nm}$ diameter were observed in the sample collected on the $\mathrm{NaBH}_{4}$-desposited slide. This suggests that there were remaining gold ions that were not completely reduced during the travel to the collector in the reducing-agent-free aqueous microdroplets. The remaining gold ions were reduced and formed observed nanoparticles with $<10$ 

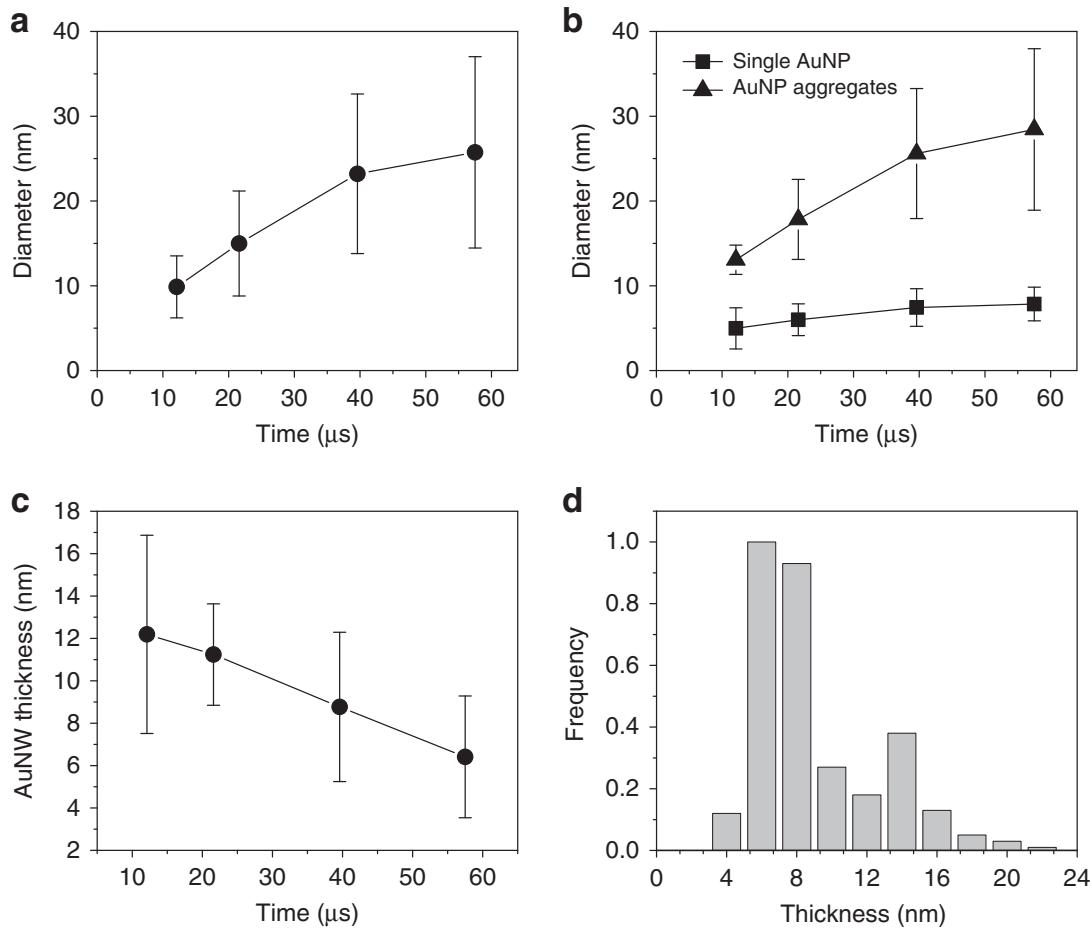

Fig. 5 Kinetics of AuNPs and AuNWs growth in the reducing-agent- and template-free microdroplets. a The diameter of AuNP aggregates as a function of time in the microdroplets. $\mathbf{b}$ The change in the diameters of individual AuNP and AuNP aggregates over time. $\mathbf{c}$ The change in the thickness of AuNW over time. $\mathbf{d}$ The bimodal distribution of the thickness of AuNWs formed in the microdroplets. Error bar represents standard deviation of the three replicates

nm diameter in a thin liquid layer with approximately several hundreds of micrometers thickness containing dissolved $\mathrm{NaBH}_{4}$ from the deposited $\mathrm{NaBH}_{4}$ on the collector. The surface effect of microdroplets would be much stronger than that of the thin liquid layer on the collector, given approximately the two order of magnitude higher surface-to-volume ratio of microdroplets compared to that of liquid layer. However, it should be noted that further reactions on the collector cannot be eliminated unless the liquid in the solution containing the unreacted gold ions is immediately desolvated upon arriving at the collector.

The separation between the three different nanostructures of nanoparticles, aggregates, and nanowires can be kinetically controlled. We have added Supplementary Fig. 9 showing the changes in the proportion of the three different types of nanostructures as a function of reaction time. At a short time of around $7.5 \mu \mathrm{s}$, the majority of the nanostructures synthesized in the microdroplets were individual nanoparticles. At $12.1 \mu \mathrm{s}$, the aggregates of individual nanoparticles and nanowires started forming. The proportion of nanowire gradually increased, while the nanoparticle aggregates reached a peak at $12.1 \mu \mathrm{s}$ and decreased. This data suggest that different types of nanostructures are formed at different temporal points; therefore, different types of gold nanostructures can be collected by controlling the microdroplet traveling time.

\section{Discussion}

We have observed that the formation of AuNPs in the microdroplets accelerated by five orders of magnitude compared to bulk, when $\mathrm{HAuCl}_{4}$ and $\mathrm{NaBH}_{4}$ are used as precursors. We also found that AuNPs are formed spontaneously from spraying an aqueous solution of $\mathrm{HAuCl}_{4}$ in the absence of a reducing agent. In addition, the nanowires and the nanowire networks are formed in these aqueous microdroplets without the use of templates or capping agents, as well as externally applied charges.
We found that by fusing streams of $\mathrm{HAuCl}_{4}$ and $\mathrm{NaBH}_{4}$ particles as large as $\sim 3 \mathrm{~nm}$ were formed only within $12 \mu \mathrm{s}$, indicating that nucleation events occur within this timescale. The particles grew up to $\sim 7 \mathrm{~nm}$ in diameter within $60 \mu \mathrm{s}$. The formation of AuNPs follow fast nucleation and slower growth mechanism, as observed in bulk by Emmerling and co-workers ${ }^{34}$. In contrast to the bulk reaction, the growth rate is increased by a factor of $1.2 \times$ $10^{5}$ and the equilibrium particle diameter is 2.1 times higher, although the concentration of the reagents used in the present study is five times less.

The accelerated formation of AuNPs in the microdroplets is consistent with other reports of reaction acceleration and catalytic behavior of microdroplets published in the last decade by us, as well as other groups ${ }^{13-16,19-25}$. However, this is the first report on accelerated crystallization in the microdroplets, which is fundamentally different from the bimolecular reactions studied previously. We speculate that the mechanism of enhanced reaction rate in the microdroplets stems from a combination of factors, mostly from air-water interface effects: surface localization of reactants, facilitated diffusion, self-alignment or organization of reactants, inhomogeneous distribution of dissociated ions, and the strong electric field at the surface of the microdroplet ${ }^{27,45,46}$. These combined effects might accelerate the AuNP formation in the microdroplets by either increasing the collision frequency of the precursors or lowering the entropic barriers of reactions.

It is surprising that AuNPs are also formed in the microdroplets without the use of any reducing agent or externally applied charge. Although we observe individual nanoparticles, we also observe aggregates, which can be attributed to the absence of a stabilizing agents. From the kinetic data presented in Figs. 4 and 5 , it can be seen that gradual aggregation of the nanoparticles occurs with progression in time. However, it seems the aggregates reach a stable size of $\sim 30 \mathrm{~nm}$ in diameter. We are not able to elucidate completely the mechanism of AuNP formation under these conditions, although some characterization has been made in the present studies. We confirmed the reduction of gold ions at 
a

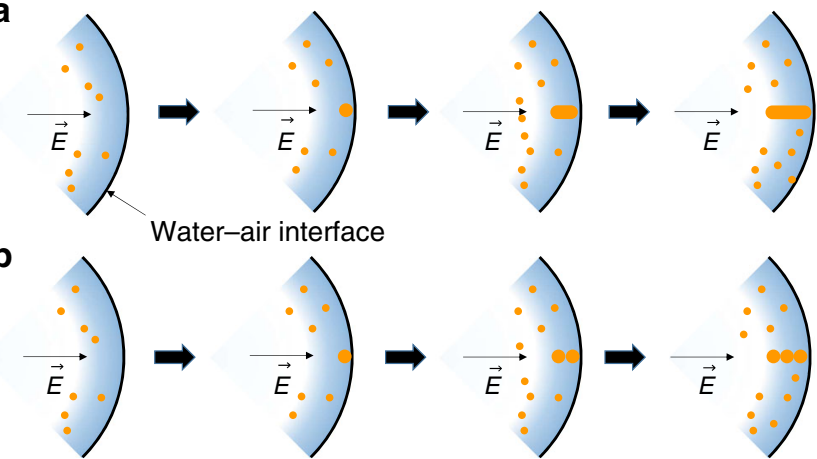

Fig. 6 Possible mechanism for nanowire growth in the microdroplets. a AuNW formation by the growth of an initial seed at the surface of the microdroplet. b AuNW grown by the self-assembly of AuNPs

molecular level by examining the mass spectrum of $\mathrm{HAuCl}_{4}$ solutions in the microdroplets. The gold ions with reduced oxidation number of +1 and +1.5 were observed, compared to the original oxidation number of +3 for $\mathrm{AuCl}_{4}$ ion. Further details on the mechanism of the spontaneous reduction are being investigated currently in our laboratory and will be presented in the follow-up publication.

Prior studies have shown that electric fields as high as $8.9 \times 10^{7}$ $\mathrm{V} \mathrm{m}^{-1}$ exist across the air-water interface ${ }^{47}$. It has been reported that ions are distributed nonuniformly throughout the aqueous microdroplets. The electric field strength can be much enhanced if the charge separation distance reduces. This electric field strength can be further increased by fluctuations occurring at the microdroplet surface, which can be induced by air-water friction or capillary waves ${ }^{48}$. These large electric fields may promote the reduction of $\mathrm{AuCl}_{4}^{-}$to Au. Alternatively, it has also been shown that the surface of water is rich in hydroxyl ions. Considering the redox potential of $\mathrm{AuCl}_{4}^{-}+2 \mathrm{e}^{-} \rightarrow \mathrm{AuCl}_{2}{ }^{-}+2 \mathrm{Cl}^{-}$is $+0.926 \mathrm{~V}$ and that of $\mathrm{O}_{2}+\mathrm{H}_{2} \mathrm{O}+4 \mathrm{e}^{-} \rightarrow 4 \mathrm{OH}^{-}$is $+0.4 \mathrm{~V}$, it is possible that hydroxyl ions of water are oxidized, reducing $\mathrm{AuCl}_{4}{ }^{-}$in the process.

We have performed experiments to capture any oxygen species evolved during the nanostructure formation in the microdroplets. We have used tris $\left(2,2^{\prime}\right.$-bipyridyl) dichlororuthenium (II) cation $\left(\mathrm{Ru}(\mathrm{bpy})_{3}{ }^{2+}\right)$, which is water soluble and widely used for capturing the dissolved oxygen in liquids ${ }^{49,50}$. Supplementary Figure 10a shows a mass spectrum of the microdroplets containing $10 \mathrm{nM} \mathrm{Ru}(\mathrm{bpy})_{3}{ }^{2+}$. Dried $\mathrm{N}_{2}$ nebulizing gas was used to minimize the exposure of the microdroplet surface to oxygen in air. A peak of $\mathrm{Ru}(\mathrm{bpy})_{3}{ }^{2+}$ with $\mathrm{Cl}^{-}$adduct was observed at $m / z 605.08$. To examine whether the exchange of the surrounding gas to $\mathrm{O}_{2}$ would affect the oxidation of $\mathrm{Ru}(\mathrm{bpy})_{3}{ }^{2+}$, the nebulizing gas was changed to $\mathrm{O}_{2}$. No oxygenated species were observed after these changes (Supplementary Fig. 10b). Next, we recorded the mass spectrum of the microdroplets containing $10 \mathrm{nM} \mathrm{Ru}(\mathrm{bpy})_{3}{ }^{2+}$ and $50 \mu \mathrm{M} \mathrm{HAuCl}_{4}$. An oxygenated $\mathrm{Ru}(\mathrm{bpy})_{3}$ species was detected at $\mathrm{m} / z$ 585.10, which was suggested to be $\mathrm{Ru}(\mathrm{bpy})_{2}(\mathrm{bpyO})^{51}$. All the procedures were conducted in a dark room to prevent any lightinduced oxidation or decomposition of reagents. These data show that oxygen evolves during the reduction of gold ions and nanostructure formation in the microdroplets and support our argument that hydroxyl ions of water are oxidized to reduce gold ions. The oxygenated $\mathrm{Ru}(\mathrm{bpy})_{3}$ species was observed only when $\mathrm{HAuCl}_{4}$ was added to the microdroplets. This is because oxygen evolves through electron transfer only when a participating redox pair is present.

A third possibility is reductive elimination of chlorine gas. However, the reaction $\mathrm{AuCl}_{4}{ }^{-} \rightarrow \mathrm{AuCl}_{2}{ }^{-}+\mathrm{Cl}_{2}$ is endergonic $\left(>12.6 \mathrm{kcal} \mathrm{mol}^{-1}\right)$ in bulk ${ }^{52}$. It remains to be investigated whether the free-energy change in the microdroplet environment accounts for what we have observed. We have confirmed that for some reactions, the microdroplets do change the free energy of the reaction and facilitate the otherwise unfavorable reactions ${ }^{53}$.

The electric field also appears to be involved in the nanowire formation in the microdroplets. Figure 6 presents a possible mechanism in which the nanowires can be formed at the water-air interface. The nonuniformly distributed ions can exert a high electric field at the water-air interface. Several studies have reported electric-field-induced nanostrucure alignment or anisotropic growth ${ }^{54-56}$. The electric field that is orthogonal to the microdroplet surface may direct the growth of the Au nanostructure from the microdroplet surface to the center of the microdroplets. As growth of the initial nanowire occurs, the formed nanowire can further increase the charge accumulation at the tip of the nanowire that can promote growth at the tip of the nanowire (Fig. 6a). It is also possible that the nanowire is grown by collision of the nucleus or seed nanoparticles at the terminal of the initially grown nanowire (Fig. 6b). From the Figs. 4 and 5, it can be deduced that initially the AuNWs are grown by the mechanism proposed in Fig. 6b and followed by Fig. 6a. Based on the kinetic data (Figs. 4 and 5), we propose the following mechanism for the formation of AuNWs. We observe that the width of the AuNWs are more or less invariant with time and is similar to the diameter of the AuNPs formed, although the length of the wires increases. From Fig. 4, it is clear that the AuNPs formed a self-assembly to form some AuNWs. Self-assembly of AuNPs into AuNWs have been observed before. For example, Bjørnholm et al. demonstrated the formation of $2 \mathrm{D}$ AuNWs at the air-water interface by using surfactants ${ }^{57}$. Rajh et al. have shown the self-assembly of the charged AuNPs on exposure to electron beam ${ }^{58}$. The present work is a striking demonstration of self-assembly of nanoparticles without the use of any surfactants or externally applied strong fields.

We have studied the formation of gold nanostructures in the aqueous microdroplets traveling through air at room temperature. We have found that the reaction rate is enhanced by many orders of magnitude, compared to bulk solution. We have also discovered an unexpected and new phenomenon: spontaneous formation of AuNPs, as well as nanowires in the absence of any reducing agent or template. Although not completely understood, we have suggested three possible mechanisms for this reaction. Our results indicate that the AuNWs are formed by self-assembly of the nanoparticles. It should be noted that this is the first demonstration where self-assembly can be obtained without the use of surfactants or externally applied strong fields. Our results demonstrate that microdroplets have a unique environment and hold the potential to be used as powerful microreactors for the synthesis and template-free control of nanostructures.

\section{Methods}

Chemicals. Gold(III) chloride trihydrate $\left(\mathrm{HAuCl}_{4} \cdot 3 \mathrm{H}_{2} \mathrm{O}, 520918\right), \mathrm{NaBH}_{4}$, and tris (2,2-bipyridyl)dichlororuthenium(II) hexahydrate were purchased from SigmaAldrich (St. Louis, MO). HPLC-grade water were purchased from Fisher Scientific (Nepean, ON, Canada).

Synthesis of AuNPs using microdroplet fusion. We used the microdroplet fusion setup for kinetically controlled growth of AuNPs. The microdroplet fusion was conducted as previously described ${ }^{13}$. Briefly, two streams of the microdroplets were generated by a high-pressure, dry $\mathrm{N}_{2}$ gas at 120 psi. The two microdroplet sources are equipped with an $\mathrm{X}-\mathrm{Y}-\mathrm{Z}$ micro-positioning linear and angular stage for accurate alignment of the two microdroplet streams. This alignment is important for ensuring fusion of most of the incident droplets and for maintaining a linear trajectory toward the collector. The best alignment was acquired with the angle between two crossed microdroplet streams at $78^{\circ}$, which showed the highest probability of droplet fusion and straight trajectories of the fused microdroplets to the inlet of the mass spectrometer. Two solutions of analytes $\left(\mathrm{HAuCl}_{4}\right.$ solution at 
$100 \mu \mathrm{M}$ and $\mathrm{NaBH}_{4}$ at $\left.400 \mu \mathrm{M}\right)$ were injected from the two microdroplet sources with a syringe pump (Harvard Apparatus, Holliston, MA) at a flow rate of $30 \mu \mathrm{L}$ $\mathrm{min}^{-1}$. The stream of fused microdroplets at a speed of $83 \mathrm{~m} \mathrm{~s}^{-1}$, which was previously measured in the charged microdroplets using a high-speed camera and particle tracking ${ }^{13}$, was collected on glass slides. This speed is assumed to be similar to that of the uncharged microdroplets used in the present studies. This reason is because the predominant factor affecting the velocity of the microdroplets is the nebulizing gas pressure, rather than the Coulomb force between the source of the microdroplets and the counter electrode. The collected fused microdroplets containing reaction products were transferred to a TEM grid for TEM imaging. The samples were dehydrated completely prior to being mounted onto the TEM holder. The sample drying was carried out in open air or in a vacuum desiccator. The approximate drying durations were $30 \mathrm{~min}$ in open air and $5 \mathrm{~min}$ in a vacuum desiccator.

To examine whether any further reaction can occur during the collection on a glass slide, the experiments of spraying the fused microdroplets on $\mathrm{NaBH}_{4}$ deposited glass slides was conducted. The stream of the fused microdroplets generated by fusing the microdroplets containing $100 \mu \mathrm{M} \mathrm{HAuCl}_{4}$ solution in water and the microdroplets containing $400 \mu \mathrm{M} \mathrm{NaBH}_{4}$ solution in water were sprayed on the $\mathrm{NaBH}_{4}$-deposited glass slides. Separate experiments of spraying the fused microdroplets were carried out by fusing the microdroplets containing 100 $\mu \mathrm{M} \mathrm{HAuCl}{ }_{4}$ and the microdroplets with no regents and then sprayed on the $\mathrm{NaBH}_{4}$-deposited glass slides. The traveling distance of the fused microdroplets were maintained at $15 \mathrm{~mm}$ for both sets of experiments. The samples collected on the $\mathrm{NaBH}_{4}$-deposited glass slides were transfer to a TEM grid for imaging.

The kinetics of the formation of different nanostructures including individual nanoparticles, aggregates of nanoparticles, and nanowires were measured by increasing the traveling distance of the fused microdroplets. The samples collected on glass slides with different traveling time points of 7.5, 12.1, 21.6, 39.6, and 57.5 $\mu \mathrm{s}$ were transferred to the TEM grid for TEM analysis.

Characterization of nanostructures. TEM imaging was carried out using FEI Tecnai G2 F20 X-TWIN Transmission Electron Microscope operated at $200 \mathrm{keV}$ and FEI Titan TEM for a high-resolution TEM imaging operated at $300 \mathrm{keV}$. Three different types of TEM grids made of ultrathin carbon film on lacey carbon support film, carbon film on copper substrate, and carbon on gold substrate (Ted Pella, Redding, CA) were used for TEM imaging. EDS analysis was performed with an EDS detector on the Tecnai TEM. The presence of nanostructures produced in the microdroplets was also examined by dynamic light scattering (DLS). A $100 \mu \mathrm{L}$ $\mathrm{HAuCl}_{4}$ solution was sprayed into a $20 \mathrm{~mL}$ vial for $30 \mathrm{~min}$. The resultant sample was diluted with $1 \mathrm{~mL}$ water used for DLS measurement. The difference in the frequency distribution between the control sample and the nanoparticle solution was measured.

Mass spectrometry analysis. Thermo Scientific LTQ Orbitrap XL Hybrid Ion Trap-Orbitrap mass spectrometer was used for mass spectrometric analysis. $\mathrm{HAuCl}_{4}$ solution at $10 \mu \mathrm{m}$ concentration prepared in $\mathrm{H}_{2} \mathrm{O}$ was sprayed with highpressure dry $\mathrm{N}_{2}$ gas at $120 \mathrm{psi}$ to generate a stream of microdroplets. No voltage was applied to the solution. The heated capillary inlet of the mass spectrometer was maintained at $\sim 275^{\circ} \mathrm{C}$. The capillary temperature was decreased to $60^{\circ} \mathrm{C}$ to examine whether a thermal decomposition of the ions occurred in the capillary. The capillary and tube lens voltages were set as $-44 \mathrm{~V}$ and $-60 \mathrm{~V}$.

Capturing oxygen evolution during nanostructure formation in the microdroplets was carried out by using oxygen-binding molecule tris $\left(2,2^{\prime}\right.$ bipyridyl) dichlororuthenium (II) cation $\left(\mathrm{Ru}(\mathrm{bpy})_{3}{ }^{2+}\right)$. Microdroplets containing $10 \mathrm{nM} \mathrm{Ru}(\mathrm{bpy})_{3}{ }^{2+}$ and $50 \mu \mathrm{M} \mathrm{HAuCl}_{4}$ was sprayed into the mass spectrometer inlet with $100 \% \mathrm{~N}_{2}$ nebulizing gas. As a control experiment, a mass spectrum of the microdroplets containing only $10 \mathrm{nM} \mathrm{Ru}(\mathrm{bpy})_{3}{ }^{2+}$ was recorded with $100 \% \mathrm{~N}_{2}$ nebulizing gas to ensure that $\mathrm{Ru}(\mathrm{bpy})_{3}{ }^{2+}$ was not contaminated. To examine whether the oxidation of $\mathrm{Ru}(\mathrm{bpy})_{3}$ was induced by oxygen in the air, the nebulization gas was replaced with $100 \%$ oxygen gas. The mass spectrum for the samples with $\mathrm{Ru}(\mathrm{bpy})_{3}{ }^{2+}$ were recorded in positive mode. The heated capillary inlet to the mass spectrometer was maintained at $\sim 275^{\circ} \mathrm{C}$. The capillary and tube lens voltages were set as $-44 \mathrm{~V}$ and $-60 \mathrm{~V}$.

Data availability. The authors declare that the data supporting the findings of this study are available in this article and its Supplementary Information Files, or from the corresponding authors on request.

Received: 5 October 2017 Accepted: 28 March 2018

Published online: 19 April 2018

\section{References}

1. Barrow, S. J., Wei, X., Baldauf, J. S., Funston, A. M. \& Mulvaney, P. The surface plasmon modes of self-assembled gold nanocrystals. Nat. Commun. 3, 1275 (2012).
2. Daniel, M.-C. \& Astruc, D. Gold nanoparticles: assembly, supramolecular chemistry, quantum-size-related properties, and applications toward biology, catalysis, and nanotechnology. Chem. Rev. 104, 293-346 (2004).

3. Elghanian, R., Storhoff, J. J., Mucic, R. C., Letsinger, R. L. \& Mirkin, C. A. Selective colorimetric detection of polynucleotides based on the distancedependent optical properties of gold nanoparticles. Science 277, 1078-1081 (1997).

4. Mirkin, C. A., Letsinger, R. L., Mucic, R. C. \& Storhoff, J. J. A DNA-based method for rationally assembling nanoparticles into macroscopic materials. Nature 382, 607-609 (1996).

5. Turner, M. et al. Selective oxidation with dioxygen by gold nanoparticle catalysts derived from 55-atom clusters. Nature 454, 981 (2008).

6. Ciganda, R. et al. Liquid-liquid interfacial electron transfer from ferrocene to gold (III): an ultrasimple and ultrafast gold nanoparticle synthesis in water under ambient conditions. Inorg. Chem. 55, 6361-6363 (2016).

7. Leng, W., Pati, P. \& Vikesland, P. J. Room temperature seed mediated growth of gold nanoparticles: mechanistic investigations and life cycle assesment. Environ. Sci.: Nano 2, 440-453 (2015).

8. Sajanlal, P. R., Sreeprasad, T. S., Samal, A. K. \& Pradeep, T. Anisotropic nanomaterials: structure, growth, assembly, and functions. Nano Rev. 2, 5883 (2011).

9. Sun, Y. \& Xia, Y. Shape-controlled synthesis of gold and silver nanoparticles. Science 298, 2176-2179 (2002).

10. An, K. \& Somorjai, G. A. Size and shape control of metal nanoparticles for reaction selectivity in catalysis. ChemCatChem 4, 1512-1524 (2012).

11. Tao, A. R., Habas, S. \& Yang, P. Shape control of colloidal metal nanocrystals. Small 4, 310-325 (2008).

12. Huang, X. et al. Synthesis of hexagonal close-packed gold nanostructures. Nat. Commun. 2, 292 (2011).

13. Lee, J. K., Kim, S., Nam, H. G. \& Zare, R. N. Microdroplet fusion mass spectrometry for fast reaction kinetics. Proc. Natl Acad. Sci. USA 112, 3898-3903 (2015)

14. Lee, J. K., Nam, H. G. \& Zare, R. N. Microdroplet fusion mass spectrometry: accelerated kinetics of acid-induced chlorophyll demetallation. Q. Rev. Biophys. 50, e2 (2017).

15. Lee, J. K., Banerjee, S., Nam, H. G. \& Zare, R. N. Acceleration of reaction in charged microdroplets. Q. Rev. Biophys. 48, 437-444 (2015).

16. Banerjee, S. \& Zare, R. N. Syntheses of isoquinoline and substituted quinolines in charged microdroplets. Angew. Chem. Int. Ed. 54, 14795-14799 (2015).

17. Yan, X., Cheng, H. Y. \& Zare, R. N. Two-phase reactions in microdroplets without the use of phase-transfer catalysts. Angew. Chem. Int. Ed. 56, 3562-3565 (2017).

18. Jansson, E. T., Lai, Y. H., Santiago, J. G. \& Zare, R. N. Rapid hydrogendeuterium exchange in liquid droplets. J. Am. Chem. Soc. 139, 6851-6854 (2017).

19. Girod, M., Moyano, E., Campbell, D. I. \& Cooks, R. G. Accelerated bimolecular reactions in microdroplets studied by desorption electrospray ionization mass spectrometry. Chem. Sci. 2, 501-510 (2011).

20. Müller, T., Badu-Tawiah, A. \& Cooks, R. G. Accelerated carbon-carbon bondforming reactions in preparative electrospray. Angew. Chem. Int. Ed. 51, 11832-11835 (2012).

21. Badu-Tawiah, A., Campbell, D. \& Cooks, R. G. Accelerated C-N bond formation in dropcast thin films on ambient surfaces. J. Am. Soc. Mass Spectrom. 23, 1461-1468 (2012).

22. Bain, R. M., Pulliam, C. J. \& Cooks, R. G. Accelerated Hantzsch electrospray synthesis with temporal control of reaction intermediates. Chem. Sci. 6, 397-401 (2015).

23. Bain, R. M., Pulliam, C. J., Ayrton, S. T., Bain, K. \& Cooks, R. G. Accelerated hydrazone formation in charged microdroplets. Rapid Commun. Mass Spectrom. 30, 1875-1878 (2016).

24. Yang, J., Qi, L., Qiao, J., Chen, Y. \& Ma, H. Rate acceleration of the BaylisHillman reaction within microreactors. Chin. J. Chem. 29, 2385-2388 (2011).

25. Li, Y., Yan, X. \& Cooks, R. G. The role of the interface in thin film and droplet accelerated reactions studied by competitive substituent effects. Angew. Chem. Int. Ed. 55, 3433-3437 (2016).

26. Bain, R. M., Pulliam, C. J., Thery, F. \& Cooks, R. G. Accelerated chemical reactions and organic synthesis in leidenfrost droplets. Angew. Chem. Int. Ed. 55, 10478-10482 (2016).

27. Fallah-Araghi, A. et al. Enhanced chemical synthesis at soft interfaces: a universal reaction-adsorption mechanism in microcompartments. Phys. Rev. Lett. 112, 028301 (2014).

28. Nakamura H. Nanoparticle synthesis in microreactors. Encyclopedia of Microfluidics and Nanofluidics (Springer, New York, 2008).

29. Rahman, M. T. \& Rebrov, E. V. Microreactors for gold nanoparticles synthesis: from Faraday to flow. Processes 2, 466-493 (2014).

30. Cottam, B. F., Krishnadasan, S., deMello, A. J., deMello, J. C. \& Shaffer, M. S. P. Accelerated synthesis of titanium oxide nanostructures using microfluidic chips. Lab Chip 7, 167-169 (2007). 
31. Li, A. Y., Luo, Q. J., Park, S. J. \& Cooks, R. G. Synthesis and catalytic reactions of nanoparticles formed by electrospray ionization of coinage metals. Angew. Chem. Int. Ed. 53, 3147-3150 (2014).

32. Deraedt, C. et al. Sodium borohydride stabilizes very active gold nanoparticle catalysts. Chem. Commun. 50, 14194-14196 (2014).

33. Lin, C., Tao, K., Hua, D., Ma, Z. \& Zhou, S. Size effect of gold nanoparticles in catalytic reduction of p-nitrophenol with NaBH4. Molecules 18, 12609-12620 (2013).

34. Polte, J. et al. Nucleation and growth of gold nanoparticles studied via in situ small angle X-ray scattering at millisecond time resolution. ACS Nano 4, 1076-1082 (2010).

35. Kojima, Y. \& Kato, T. Nanoparticle formation in Au thin films by electronbeam-induced dewetting. Nanotechnology 19, 255605 (2008).

36. Gong, J. et al. In-situ synthesis of Ag nanoparticles by electron beam irradiation. Mater. Charact. 110, 1-4 (2015).

37. Zheng, H. et al. Observation of single colloidal platinum nanocrystal growth trajectories. Science 324, 1309-1312 (2009).

38. Otto, K., Acik, I. O., Krunks, M., Tõnsuaadu, K. \& Mere, A. Thermal decomposition study of $\mathrm{HAuCl} 4 \cdot 3 \mathrm{H} 2 \mathrm{O}$ and $\mathrm{AgNO} 3$ as precursors for plasmonic metal nanoparticles. J. Therm. Anal. Calorim. 118, 1065-1072 (2014).

39. Yoo, Y. et al. Steering epitaxial alignment of $\mathrm{Au}, \mathrm{Pd}$, and AuPd nanowire arrays by atom flux change. Nano Lett. 10, 432-438 (2010).

40. Forrer, P., Schlottig, F., Siegenthaler, H. \& Textor, M. Electrochemical preparation and surface properties of gold nanowire arrays formed by the template technique. J. Appl. Electrochem. 30, 533-541 (2000).

41. Jana, N. R., Gearheart, L. \& Murphy, C. J. Wet chemical synthesis of high aspect ratio cylindrical gold nanorods. J. Phys. Chem. B 105, 4065-4067 (2001).

42. Xia, Y. et al. One-dimensional nanostructures: synthesis, characterization, and applications. Adv. Mater. 15, 353-389 (2003).

43. Huang, Y., Fang, Y., Zhang, Z., Zhu, L. \& Sun, M. Nanowire-supported plasmonic waveguide for remote excitation of surface-enhanced Raman scattering. Light: Sci. Appl. 3, e199 (2014).

44. Jakhmola A., et al. Self-assembly of gold nanowire networks into gold foams: production, ultrastructure and applications. Inorganic Chemistry Frontiers 4, 1033-1041 (2017).

45. Jung, Y. \& Marcus, R. On the theory of organic catalysis "on water". J. Am. Chem. Soc. 129, 5492-5502 (2007).

46. Narayan, S. et al. "On water": Unique reactivity of organic compounds in aqueous suspension. Angew. Chem. Int. Ed. 44, 3275-3279 (2005).

47. Kathmann, S. M., Kuo, I.-F. W. \& Mundy, C. J. Electronic effects on the surface potential at the vapor-liquid interface of water. J. Am. Chem. Soc. 130, 16556-16561 (2008).

48. Sutton, M. A review of X-ray intensity fluctuation spectroscopy. C. R. Phys. 9, 657-667 (2008).

49. Zhang, L., Tsow, F., Forzani, E. \& Tao, N. Reversible oxygen gas sensor based on electrochemiluminescence. Chem. Commun. 46, 3333-3335 (2010).

50. Payra, P. \& Dutta, P. K. Development of a dissolved oxygen sensor using tris (bipyridyl) ruthenium (II) complexes entrapped in highly siliceous zeolites. Microporous Mesoporous Mater. 64, 109-118 (2003).

51. Bergman, N., Thapper, A., Styring, S., Bergquist, J. \& Shevchenko, D. Quantitative determination of the Ru (bpy) $32+$ cation in photochemical reactions by matrix-assisted laser desorption/ionization time-of-flight mass spectrometry. Anal. Methods 6, 8513-8518 (2014).

52. Đurović, M. D., Puchta, R., Bugarčić, Ž. D. \& van Eldik, R. Studies on the reactions of $[\mathrm{AuCl} 4]$ - with different nucleophiles in aqueous solution. Dalton Trans. 43, 8620-8632 (2014).

53. Nam, I., Lee, J. K., Nam, H. G. \& Zare, R. N. Abiotic production of sugar phosphates and uridine ribonudeoside in aqueous microdroplets. Proc. Natl Acad. Sci. USA 114, 12396-12400 (2017).
54. Ahmed, W., Kooij, E. S., Van Silfhout, A. \& Poelsema, B. Quantitative analysis of gold nanorod alignment after electric field-assisted deposition. Nano Lett. 9 , 3786-3794 (2009)

55. Cheng, C., Gonela, R. K., Gu, Q. \& Haynie, D. T. Self-assembly of metallic nanowires from aqueous solution. Nano Lett. 5, 175-178 (2005).

56. Chen, C.-Y. et al. Liquid-solid process for growing gold nanowires on an indium tin oxide substrate as excellent field emitters. J. Phys. Chem. C 116, 12824-12828 (2012)

57. Hassenkam, T. et al. Fabrication of 2D gold nanowires by self-assembly of gold nanoparticles on water surfaces in the presence of surfactants. $A d v$. Mater. 14, 1126-1130 (2002).

58. Maurer, J. H., González-García, L., Reiser, B., Kanelidis, I. \& Kraus, T. Templated self-assembly of ultrathin gold nanowires by nanoimprinting for transparent flexible electronics. Nano Lett. 16, 2921-2925 (2016).

\section{Acknowledgements}

This work was supported by the Air Force Office of Scientific Research through Basic Research Initiative grant (AFOSR FA9550-12-1-0400) and the Institute for Basic Science (IBS-R013-D1). D.S. thanks the Winston Chen Stanford Graduate Fellowship and the Center for Molecular Analysis and Design for funding. Part of this work was performed at the Stanford Nano Shared Facilities (SNSF), supported by the National Science Foundation under award ECCS-1542152.

\section{Author contributions}

J.K.L., H.G.N., and R.N.Z. conceived of the project. J.K.L., D.S., H.G.N., and R.N.Z. designed the experiments. J.K.L. and D.S. performed the experiments and analyzed the data. All authors contributed to the writing of the manuscript.

\section{Additional information}

Supplementary Information accompanies this paper at https://doi.org/10.1038/s41467 018-04023-z.

Competing interests: The authors declare no competing interests.

Reprints and permission information is available online at http://npg.nature.com/ reprintsandpermissions/

Publisher's note: Springer Nature remains neutral with regard to jurisdictional claims in published maps and institutional affiliations.

Open Access This article is licensed under a Creative Commons Attribution 4.0 International License, which permits use, sharing, adaptation, distribution and reproduction in any medium or format, as long as you give appropriate credit to the original author(s) and the source, provide a link to the Creative Commons license, and indicate if changes were made. The images or other third party material in this article are included in the article's Creative Commons license, unless indicated otherwise in a credit line to the material. If material is not included in the article's Creative Commons license and your intended use is not permitted by statutory regulation or exceeds the permitted use, you will need to obtain permission directly from the copyright holder. To view a copy of this license, visit http://creativecommons.org/ licenses/by/4.0/

(c) The Author(s) 2018 